\title{
A novel mutation in a family with non-erythroid variant form of acute intermittent porphyria
}

\begin{abstract}
Acute intermittent porphyria (AIP), an autosomal dominant disorder, is divided into two forms, the classical form (more than 95\%) and the non-erythroid variant form, according to erythroid porphobilinogen deaminase (PBGD) activity. In the variant form, the PBGD activity is essentially normal. Detection of presymptomatic mutation carriers relies on a DNA test. This variant form of AIP is very rare, with only nine families carrying five different mutations reported in the literature. Here we report a novel G-to-T transversion in the first position of intron 1 of the $P B G D$ gene in a family with this variant form of AIP. We also review all previously reported cases and propose an effective diagnostic approach.
\end{abstract}

Key words Acute intermittent porphyria - DNA testing . Splicing defect - DNA sequencing · Porphobilinogen deaminase $\cdot$ Porphobilinogen deaminase gene

\section{Introduction}

Acute intermittent porphyria (MIM 176000) is an autosomal dominant disorder characterized by acute attacks of neurological dysfunction with severe abdominal pain, peripheral neuropathy, hypertension, and tachycardia. It is caused by excess neurotoxin, aminolevulinic acid (ALA) and porphobilinogen (PBG), due to half-normal enzyme activity of PBGD. The acute attacks usually begin after puberty and are often precipitated by the usage of drugs, alcohol, and estrogen. In the classical form of AIP,

S. $\mathrm{Yu}^{1}(\bowtie) \cdot$ V. Poulos $\cdot$ P. Stewart

Department of Clinical Biochemistry, Royal Prince Alfred Hospital, Camperdown, NSW, 2050, Australia

Present address:

${ }^{1}$ Department of Cytogenetics and Molecular Genetics, Women's and Children's Hospital, 72 King William Road, North Adelaide, SA

5006, Australia

Tel. +61-8-820-47110; Fax +61-8-8204-7342

e-mail: yus@mail.wch.sa.gov.au quantitation of erythroid PBGD activity is used routinely to detect presymptomatic carriers, who demonstrate halfnormal enzyme activity. However, in the non-erythroid variant form, the PBGD activity is normal. Molecular analysis reveals that, in most cases, splicing defects in the $P B G D$ gene cause the variant form of AIP. DNA test is the only reliable means of identifying presymptomatic carriers in the affected families. We studied a family with the nonerythroid variant form of AIP for mutation in the PBGD gene, with the aim of detecting presymptomatic carriers in this family.

\section{Patients and methods}

The propositus is Caucasian and is living in New South Wales, Australia. He was diagnosed as having AIP in 1966 (age 23 years), after a first attack induced by phenytoin therapy. The diagnosis of AIP was made on the bases of clinical features typical of AIP, including acute abdominal pain, vomiting, and constipation, in combination with increased urinary ALA and PBG. However, the erythroid PBGD activity was normal. Three other family members from two generations are also affected and have had acute attacks. Erythroid PBGD activity was within the normal range in all members of the family, with no difference in this activity between those affected and those not affected. All individuals gave their informed consent prior to their inclusion in this study.

Genomic DNA was isolated from peripheral blood lymphocytes. Considering the fact that all five known mutations causing this variant form of AIP were clustered in a small DNA region, we chose to sequence this region directly. Primers AIP $3\left(5^{\prime}\right.$-acg tgt ccc cgg tac tcg c- $\left.3^{\prime}\right)$ and AIP $2\left(5^{\prime}-\right.$ atc gct gca cgg ctc gtc ca-3') were designed to amplify a fragment containing the initiation codon and the exon 1/ intron 1 junction of the $P B G D$ gene. The amplicons were purified using the Wizard PCR (polymerase chain reaction) Preps DNA Purification system for Rapid Purification of DNA fragments (Promega-Biotech, Madison, WI, USA), 


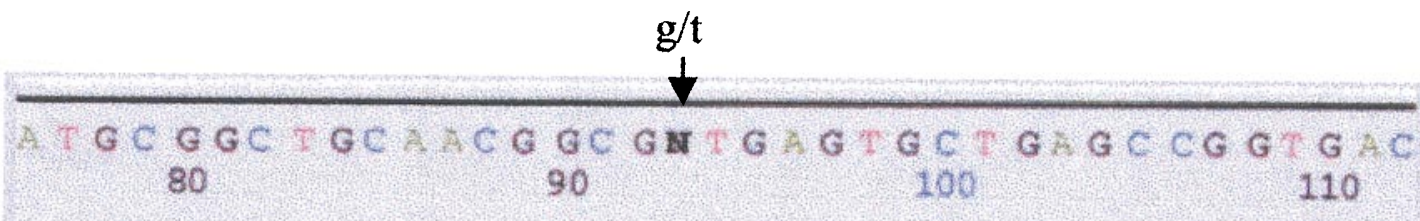

\section{AIP patient}

\section{Normal}

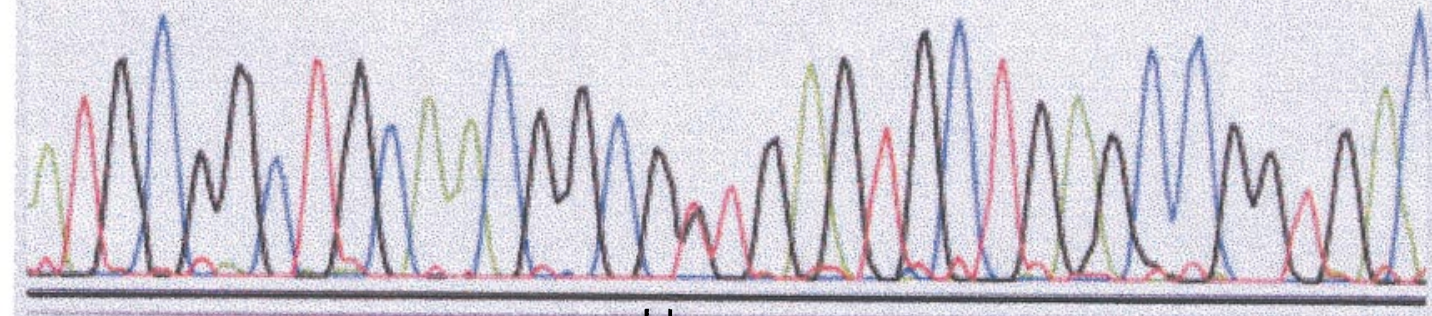

A T GCGGC GCA ACGGCGGIGAGTGCTGAGCCGGMGAC

80

$90 \quad 100$

110
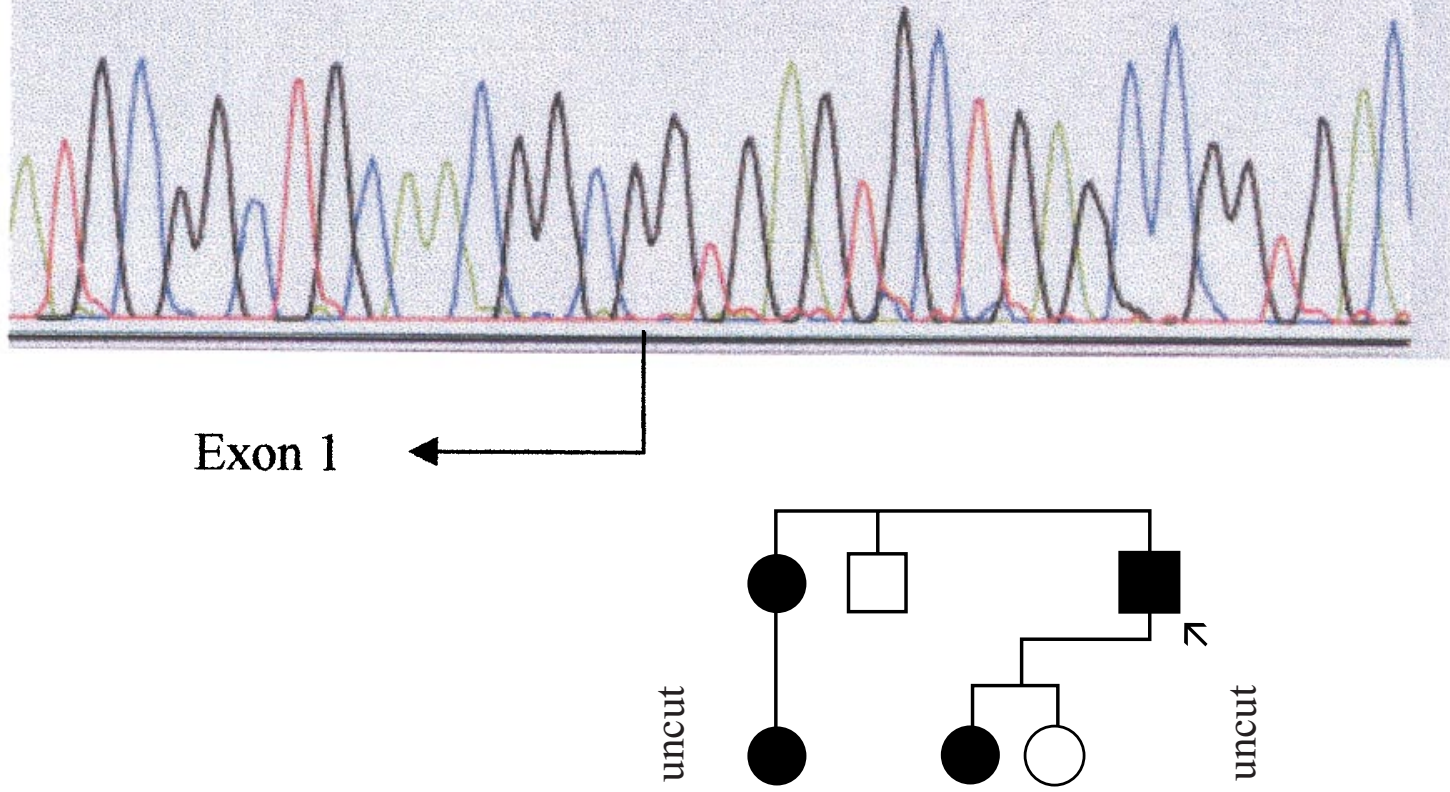

Fig. 1. A DNA sequence of the exon1/intron 1 junction of human $P B G D$ gene showing a G-to-T transversion located at the first position of intron 1 in the patient's sample. B Cosegregation of the mutation with the disorder in the family with the non-erythroid variant form of acute intermittent porphyria $(A I P)$. The sizes of the molecular weight markers are indicated on the right. The propositus is indicated with an arrow. Black symbols, affected individuals; white symbols, asymptomatic family members

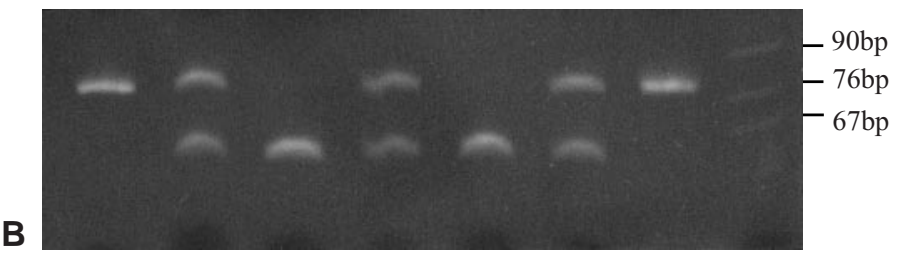

and sequenced using the dideoxy chain termination method.

Following identification of the mutation by sequencing analysis, a new primer, AIP4 (5'-gca atg cgg ctg caa ccg cg$3^{\prime}$ ), was designed to contain a $\mathrm{C}$ instead of a $\mathrm{G}$, as under-

lined in the primer sequence. This change creates a restriction enzyme KspI site (CCGCGG) in the normal sequence, but not when the mutation changes the last $\mathrm{G}$ to $\mathrm{T}$. Because AIP is an autosomal dominant disorder, the normal allele would serve as an internal control for cleavage efficiency. 
Primers AIP2 and AIP4 were used for PCR reaction. Ten $\mu \mathrm{l}$ of amplified products were digested overnight in a $20-\mu \mathrm{l}$ total volume containing 5 units of restriction enzyme $K s p \mathrm{I}$ and the matching buffer (Boehringer Mannheim, Germany). The undigested (indicated as "uncut" in Fig. 1B) and digested samples were subjected to electrophoresis in a $6 \%$ polyacrylamide gel, followed by staining of the bands with ethidium bromide. Normal individuals show only one band of 58 base pair (bp), whereas the affected individuals have two bands, of $78 \mathrm{bp}$ and $58 \mathrm{bp}$, respectively (Fig. 1B) due to abolition of the $K s p \mathrm{I}$ site by the mutation in the mutant allele.

\section{Results and discussion}

DNA sequencing identified a novel mutation, a G-to-T transversion in the first position of intron $1(33+1 \mathrm{G}-$ to-T $)$ (Fig. 1A), in two affected members of the family, but not in an asymptomatic individual from the same family. PCR amplification followed by restriction enzyme digestion showed that the mutation cosegregated with the disorder in the family (Fig. 1B). This change was not identified in 100 control chromosomes.

Previously, the human $P B G D$ gene was shown to produce two tissue-specific transcripts, erythroid and nonerythroid, initiated from two promoters of the same gene. In the classical form of AIP, both transcripts are affected. However, in the non-erythroid variant form, only the nonerythroid transcript is affected, leaving the erythroid transcript intact. Before this study, there were nine families with the variant form of AIP (one Dutch, one Finnish, one American, two German, and four French) reported to carry five disease-causing mutations in the $P B G D$ gene (Fig. 2). These include four point mutations at the exon 1/intron 1 junction of the $P B G D$ gene and one in the initiation of the translation codon of the non-erythroid transcript (Grandchamp et al. 1989a,b; Kauppinen et al. 1995; Puy et al. 1997, 1998; Chen et al. 1994). Three point mutations in the exon $1 /$ intron 1 junction were demonstrated to cause a splicing defect, leading to a 67-bp intronic sequence aberrantly transcribed and a premature stop codon (Puy et al. 1998). Because these mutations are located upstream from the promoter for the erythroid transcript, they affect only the non-erythroid transcript, resulting in half-normal PBGD activity in non-erythroid tissues. This explains why patients with the non-erythroid variant form of AIP have typical clinical and biochemical features of AIP, yet normal erythroid PBGD activity.

The mutation in this report is the sixth mutation in the $P B G D$ gene responsible for the non-erythroid variant form of AIP (Fig. 2). The novel mutation identified is located in the first position of intron 1 of the $P B G D$ gene. It is expected to cause a splicing defect in the same way as the previously described mutations. Identification of the mutation provides a simple method to detect presymptomatic carriers in the family in order to avoid acute attacks.

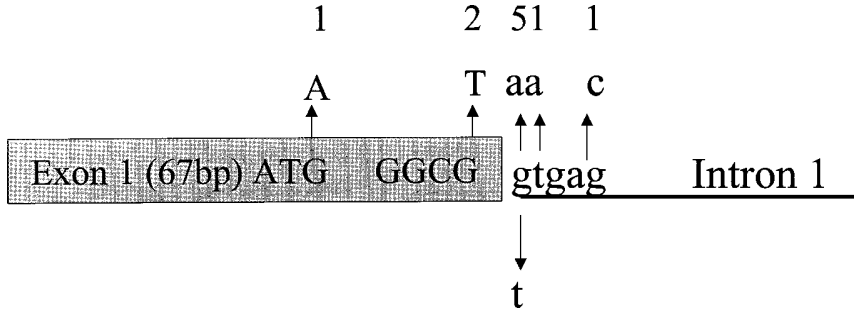

Fig. 2. Summary of known mutations in the $P B G D$ gene responsible for the non-erythroid variant form of AIP. Exon 1 sequence, in box, is shown in uppercase, with intron 1 sequences in lowercase. The length of exon 1 and the center location of the initiation codon ATG are shown. The arrows indicate the mutations identified (upward arrows for previously identified mutations and downward arrow for the mutation identified in this study). The number of families with a specific point mutation is shown above that position

Mutation detection methods used in investigating this variant form of AIP included single-strain conformation polymorphism (SSCP) analysis or denaturing gradient gel electrophoresis (DGGE) followed by DNA sequencing (Chen et al. 1994; Kauppinen et al. 1995; Puy et al. 1997, 1998). In view of the fact that all five known mutations causing this variant form of AIP were clustered in a small region of DNA (Fig. 2), we decided to directly sequence the region containing the known mutations, without using other screening methods such as SSCP or DGGE. This approach was proven to be very effective in our study, with the mutation identified in the first round of DNA sequencing without the need to optimize conditions for SSCP or DGGE. We therefore recommend that DNA sequencing of the region containing the initiation codon and the exon 1 /intron 1 junction of the $P B G D$ gene should be considered as the firstline investigation for the non-erythroid variant form of AIP.

\section{References}

Chen CH, Astrin KH, Lee G, Anderson KE, Desnick RJ (1994) Acute intermittent porphyria: identification and expression of exonic mutations in the hydroxymethylbilane synthase gene. J Clin Invest 94:1927-1937

Grandchamp B, Picat C, Mignotte V, Wilson JHP, Te Velde K, Sandkuyl L, Romeo PH, Goossens M, Nordmann Y (1989a) Tissuespecific splicing mutation in acute intermittent porphyria. Proc Natl Acad Sci USA 86:661-664

Grandchamp B, Picat C, Kauppinen R, Mignotte V, Peltonen L, Mustajoki P, Romeo PH, Goossens M, Nordmann Y (1989b) Molecular analysis of acute intermittent porphyria in a Finnish family with normal erythrocyte porphobilinogen deaminase. Eur J Clin Invest 19:415-418

Kauppinen R, Mustajoki S, Pihlaja H, Peltonen L, Mustajoki P (1995) Acute intermittent porphyria in Finland: 19 mutations in the porphobilinogen deaminase gene. Hum Mol Genet 4:215-222

Puy H, Deybach JC, Lamoril J, Robreau AM, da Silva V, Gouya L, Grandchamp B, Nordmann Y (1997) Molecular epidemiology and diagnosis of PBG deaminase gene defects in acute intermittent porphyria. Am J Hum Genet 60:1373-1383

Puy H, GroB U, Deybach JC, Robreau AM, Frank M, Nordmann Y, Doss M (1998) Exon 1 donor splice site mutations in the porphobilinogen deaminase gene in the non-erythroid variant form of acute intermittent porphyria. Hum Genet 103:570-575 